\title{
Inhabitants' Adaptive Strategies: A Case of Interactive Control in Residential Buildings
}

\author{
Vinu Subashini Rajus ${ }^{1}$, Robert Woodbury ${ }^{1}$ \\ ${ }^{1}$ School of Interactive Arts \& Technology, Simon Fraser University, Surrey, Canada
}

\begin{abstract}
Building inhabitants act more complexly than architects, engineers and designers assume. Inhabitant actions vary depending on comfort preferences, body type, clothing preference, cultural habits, knowledge of building science, controls, awareness of sustainable practices and contingencies of life. These parameters are not definitive: there might be more of which we are not aware. The more people involved, the more complexity increases. This paper summarizes inhabitants' adaptive strategies in residential buildings through a qualitative study on 14 households in Vancouver. It elucidates inhabitants' comfort preferences, barriers to sustainable adaptation and the adaptive impact of a proposed system Light Weight Look Ahead Options (LWLA).
\end{abstract}

\section{Introduction}

Designers, architects, and engineers have identified that discrepancies between predicted and actual energy usage arise with inhabitants whose action and interaction with building elements are not as simple as assumed in simulation tools or advanced user simulations. The following sections highlight a few sustainable design practices and their gaps.

\section{User models and simulations}

Advancement in technology and building controls provide more adaptive opportunities for occupants to achieve the desired comfort. Current standard models do not meet these adaptive requirements and mostly ignore the complex adaptive nature of people. The widely used Fanger PMV model is static and is more applicable to similar activities with minimal or no interactions. Post-occupancy evaluation shows that PMV progressively overestimates (De Dear (2004)) or underestimates (Becker and Paciuk (2009)) comfort perception, and ignores the dynamic daily life of inhabitants (Humphreys and Fergus Nicol (2002)). The adaptive thermal comfort model was designed to meet the dynamic nature of the inhabitants, but it is applicable only for naturally ventilated buildings. Passive design strategies are becoming the norm and buildings are retrofitted with them to improve energy efficiency. These buildings can ignore overheating and inhabitant comfort. Lomas and Porritt (2017) report that the interaction of inhabitants (especially seniors) with thermostat settings and windows in a temperate climate causes overheating in the building. Inhabitants action may cause overheating or overheating may trigger other effects. Studies of energy efficient buildings show considerable differences between assumed and actual usage (Nord et al. (2018); De Wilde (2014)). Simulation tools do not take uses such as household appliances into consideration. Further, the relationship between the "dwelling use, dwelling type, and occupant characteristics" is not well-understood (Majcen et al. (2013)). Nord et al. (2018) conclude that it is highly relevant to consider inhabitant behaviour for better prediction in Zero Energy/Emission buildings. Advances in technology have enabled access to data on occupancy and occupant energy consumption. Models of occupancy began to emerge from monitoring data and sensors. Hoes et al. (2009) conclude that simulating user behaviour is a significant parameter in energy simulations. Jia et al. (2017) classifies occupancy modelling methods (statistical, stochastic, etc.) and argues that no one model is more efficient than the others. The complexity of the model makes integration difficult and needs further study. Advanced simulations have improved and contributed to the understanding of user interaction and operations in buildings. The gaps are still significant, and designers and researchers are trying to reduce them.

\section{Home automation}

Home automation, also widely known as "smart" or "intelligent" homes, aims to help inhabitants reduce energy consumption through technologies and control strategies. This approach seems to embed the assumption that the home and system can, in and of itself, provide comfort and energy efficiency. Thus the technologies focus more on the rules of use rather than the user experience (Woodruff et al. (2007)). The actions predetermined by intelligent systems can be frustrating when they do not align with actions that the inhabitants intend (Mozer et al. (2005)). Cole and Brown (2009) argue that inhabitants have been 
neglected in smart building design, and adds inhabitant's intelligence to the five existing attributes of intelligent buildings. Recent research is shifting focus from "smart home" to "smart occupants." Such studies address inhabitants behaviour, motivational strategies and how automation can impact inhabitants' daily life and comfort (Bartram et al. (2011)). Fabi et al. (2017) highlight the importance of considering inhabitants' needs in smart buildings.

\section{System first, people second}

Designers have engaged in systems thinking, that is, understanding, modelling and configuring a design situation as an interacting assembly of parts. However, the physical parts of the building system have been given more attention than the human parts, likely because it is the physical parts that are the product of design. Designers believe that embedded systems and controls enhance inhabitant comfort, interaction, and experiences in the buildings. They largely ignore the intrusive nature of the technology; the adaptive opportunities it provides and hinders. The building design, embedded systems, and controls are judged more on the presence of physical components and performance than on its end use by inhabitants. Building simulation researchers are improving assumptions of user behaviour, but they are ignoring the engagement of inhabitants and their interaction in the buildings.

Separate from the field of design, mainly in the social sciences, researchers have developed understandings of how people, individually and in groups, actually interact with buildings. Often, for a passive design to be successful, the user needs to operate with the building elements to make the performance complete: passive design requires active inhabitants (Hampton (2011); Cole et al. (2010)). Research shows otherwise. Inhabitants' reasons for not performing an interaction at the appropriate time run the rich gamut of human experience-including the pressure of time, unawareness, lack of motivation, negligence of the inhabitants, multiple inhabitants issues, aesthetics, noise issues, and forgetfulness, to name only a few (Hampton (2011)). Understanding and designing for inhabitant engagement appears to be a sound strategy for improving performance.

\section{Engaging inhabitants}

Designing for feedback is one technique designers use to involve the inhabitants in saving energy actively (Flemming et al. (2008)). Feedback has both positive and negative impacts on inhabitant energy interventions (Abrahamse et al. (2005)). Having feedback supports energy control, but the quality of the feedback affects sustainable action outcomes (Darby (2006)). Feedback systems mostly show resource consumption and its effect but do not help the users to take the next set of actions to reduce energy consumption (Flemming et al. (2008)). Also, it is not enough to design feedback - the system needs to understand the context and the behaviour that needs to be influenced or changed (Froehlich et al. (2010)). Though feedback systems have issues to address, they still have some positive effect on inhabitants' behaviour. Faruqui et al. (2010) argue that In-Home displays encourage consumers to make efficient use of energy and can reduce consumption on an average by $7 \%$. Feedback with motivational techniques, incentives, a larger context, and social interaction improves inhabitant engagement (Strengers (2008)).

A problem with current monitoring systems is they display current energy use and predict the future based on histories. They do not explain the reason for differences in their energy consumption. Also, inhabitants may have various possibilities of actions to perform for their comfort issue based on their context. The implications of such actions are not modeled in most systems. Various studies aim to improve feedback in smart residential buildings, but they do not display the possibilities of action with comfort and energy implications. To engage and make inhabitants aware of the possible effects with comfort and energy usage we built a feedback system: Light Weight Look Ahead options (LWLA), which runs multiple parallel simulations of various possibilities with the building system and elements. LWLA aims to include detailed "lived experience" in the design of control systems for buildings. It aims to include many relevant factors, including social factors, family relationships, building type, building idiosyncrasies, cultural background, knowledge of buildings. Its design stance is that a system needs to be an aid for people not just to control their environment, but also to understand it. This paper continues the analysis presented in the SimAUD paper (Rajus and Woodbury (2018)). Here we summarize inhabitants' attributes for comfort modelling; barriers to sustainable adaptations; and the adaptive impact of the proposed system.

\section{Research design}

The research aims to understand inhabitant adaptations in buildings by designing an interactive feedback system and conducting qualitative research. The goal of LWLA was to have a deeper understanding of inhabitants' interaction in buildings. The interactive feedback system displays information on comfort and energy usage based on inhabitants interaction with building systems (like a thermostat) and elements (like windows). The qualitative research was conducted at the participants' dwelling to understand: Who they are? How do they adapt? And, how does the system assist them to adapt?

\section{Interactive system design}

The LWLA system design reifies the following ideas:

Lookahead in alternatives, context and time

LWLA provides look ahead with alternatives, context 
and time. For example, an inhabitant may be feeling cold in the building, and the reason may be because the windows or door were left open, metabolism level may be low, thermostat setting may be low, or they may be wearing light clothing. The choices may depend on social factor, personal factors, operative building elements, and systems. The novelty of LWLA system is that it will run possible alternatives related to the context and reveal its implication over time. Interaction in buildings affects the future more than the present.

\section{Multiple interaction models}

Inhabitants have three ways to express their discomfort, and these models have different expression and control freedom. The first expression is the "I feel" model. The "I feel" interaction comprises a single selection and the system responses with possible choices considering the context and building parameters. The inhabitants express their perception of feelings based on the PMV comfort scale. For example, "I am feeling cold," and the system displays the possible alternatives with comfort and energy usage (See Figure 2). "If buildings could talk" is an interaction model where the building alerts the inhabitants, if the comfort level is above or below threshold. It is automation with set criteria. Here, the inhabitants need to initiate the system to alert them based on their comfort thresholds. "What if?" model is where inhabitants can make individual inquiries. For example, what happens if they leave the windows open and at what time will it make the room uncomfortable. "What if?" model aims at exploring options. These models are explained in detail in the SimAUD paper (Rajus and Woodbury (2018)).

\section{Data comparison}

LWLA compares cost and comfort data whereas the existing monitoring systems display information based only on energy usage. The information considers two main factors. Firstly, personal factors like clothing, activities, and consumption of food or beverages. Secondly, building elements (like windows or blinds) and systems like a thermostat. The system analyses data only for thermal comfort model.

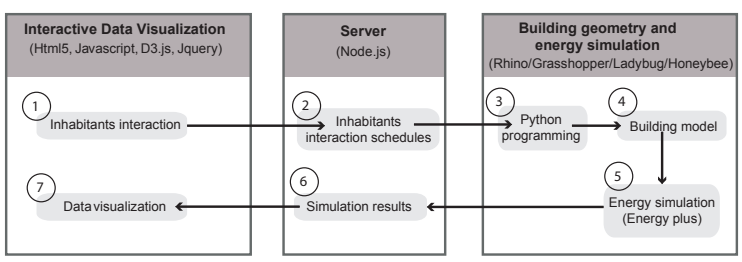

Figure 1: System Design.

Figure 1 shows the overall system design. It has three parts: interactive data visualization; server; building geometry and simulation. The interactive data visualization allows the inhabitants to interact with the three models. The server stores the interactive data of the inhabitants and triggers multiple parallel simulations. The building geometry and simulationan energy-efficient house was modeled in Rhinocerous/Grasshopper, and Ladybug/Honeybee platform runs, the energy simulation. Once the simulation runs the system updates the information. Figure 2 shows the user interface designed in HTML 5, Java script, jQuery and data visualization using D3.js. Figure 3 shows the python scripting in Grasshopper.

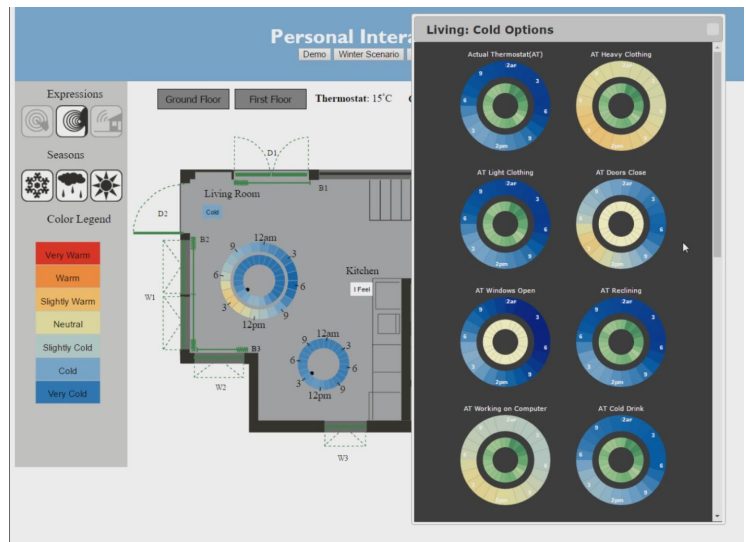

Figure 2: LWLA Interface design for "I feel" model.

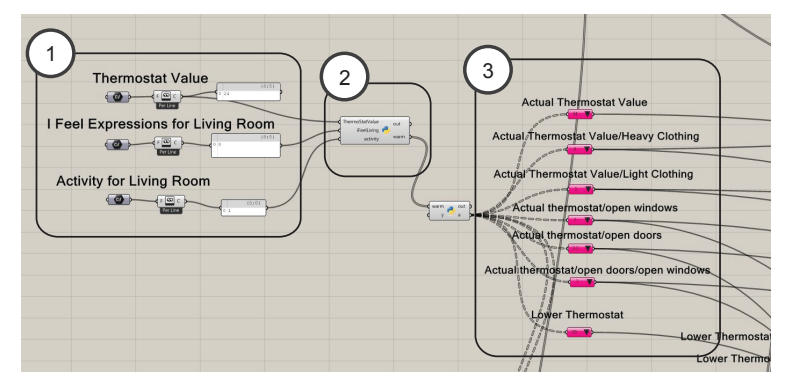

Figure 3: Screen shot of options generator in Grasshopper. (1) Input data from the server, (2) Python component, (3) Possible options.

\section{User study}

The study was conducted in the participant's house, as tasks of a few hours and over extended periods. Conducting the study at the participants' house gave clear contextual data. The participants were asked to narrate their experiences as we could not watch what they do daily over a period. The contextual study enabled the participants to explain their issues by acting them out and interacting with their residence. The data collection methods in this study were semi-structured interviews, participant observation, and field notes. No meter data was collected, the questionnaire and tasks posed different scenarios that captured their adaptive strategies.

The participants were recruited from different community groups and by the spread of word through friends and faculty. The criteria considered were age, family size and building type (like apartments or individual homes, see Figure 4). The study mainly focused on families with or without children and with or 
without seniors living with them. Children were not participants for research ethics reasons. Couples provided very different data than individual interviews. Hence, we decided to balance individuals and couples. However, conducting the study as couples gave varying responses. One person says I do this action; the other person starts to explain the conflict they have in their choice. Also, they helped each other in remembering the issues and struggles they faced in achieving comfort or understanding energy usage. A disadvantage is a personal opinion may be overpowered by the other. The study is balanced as it considers seven individual and seven couples. Sustainability involvement and awareness leaned towards a positive response to the tool. Hence, we involved a very few sustainable enthusiast. Inter-rater reliability was conducted in Nvivo 11 for three households (two couples and one individual). The data were partially coded and considered two major sections of the study: adaptations made and interaction models. It was evaluated using Kappa coefficient and percentage agreement. We found that adaptations made has a good agreement and interaction models have a moderate agreement (See Table 1).

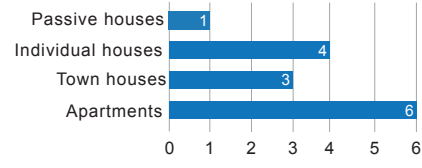

(a). Types of residential buildings

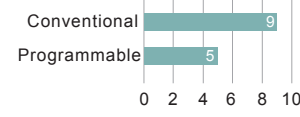

(b). Thermostat types
Figure 4: Building Demographics.

Table 1: Inter-rater reliability.

\begin{tabular}{|c|c|c|c|c|c|c|}
\hline Nodes & $2 . \mathrm{AM}^{*}$ & $2 . \mathrm{AM}^{*}$ & $2 . \mathrm{AM}^{*}$ & 3. IM* & 3. IM* & 3. IM* \\
\hline Sources & D1\&D2 & HF & IM\&IF & D1\&D2 & HF & IM\&IF \\
\hline Kappa & 0.7368 & 0.7071 & 0.7419 & 0.4591 & 0.5127 & 0.4863 \\
\hline Agrement \% & 90.21 & 93.78 & 91.84 & 90.33 & 91.05 & 93.92 \\
\hline A \& B\% & 19.75 & 8.96 & 15.43 & 5.02 & 5.69 & 3.28 \\
\hline Not A \& Not B \% & 70.46 & 84.82 & 76.41 & 85.31 & 85.36 & 90.64 \\
\hline Disagreement \% & 9.79 & 6.22 & 8.16 & 9.67 & 8.95 & 6.08 \\
\hline A \& Not B\% & 6.3 & 3.54 & 7.3 & 7.3 & 6.63 & 3.09 \\
\hline B \& Not A\% & 3.48 & 2.68 & 0.85 & 2.38 & 2.32 & 2.99 \\
\hline
\end{tabular}

AM is Adaptations Made; IM is Interaction Models.

\section{Findings}

The results show three categories: (1) inhabitants attributes for comfort preferences; (2) barriers to sustainable adaptations; and (3) adaptation through LWLA.

\section{Inhabitants' attributes for comfort modelling}

In this section, we highlight five key points that comfort modelling tools ignore.

\section{People (and households) are idiosyncratic}

"I am more a colder person, and she is a warmer person"-FM

Participants FM and FF articulate the reason for a multiple comfort model, as their family has three children and have various comfort preferences. FM says that he is colder in comparison to his spouse and he often needs to wear a sweater to be comfortable. He was not happy with the trade-off. He argues the differences in the temperature an adult chooses is higher in comparison to a child. Therefore, he suggests the need for multiple comfort personas to resolve the issues. One-size-fits-all does not work for comfort settings. Another participant LM needed solutions to the conflict they are facing with temperature settings every day. It is a challenge to consider multiple occupants with varying body temperature and personalities for energy modelling. But the problem needs to be approached. Out of the seven couple and seven individual studies, only one couple mentioned their similarities in comfort preferences.

\section{People carry cultural adaptive knowledge}

"It is ingrained in you"-HF

A person relocating comes with habits, beliefs, and practices from their region. The weather and adaptations vary between countries, within countries, and within states. Two couples who moved from Mexico (and other warm countries) to cold Vancouver revealed that cultural practices prevail. During cold winter they said they would wear sweaters. One of the reason was back home in their country they do not have a heater. So, whenever they felt cold, they would wear a sweater. It is a good sustainability practice. Contrarily, a participant narrated how her friend wears light clothes and flip-flops during winter. She cranks her thermostat to be comfortable. This person is from the Philippines and is comfortable wearing light clothes. Similarly, a couple who moved from South Africa mentions they like fresh air and the room to be cooler hence they leave the windows and heat on. In both scenarios, the building loses energy.

Acclimatization takes time, and it influences adaptive behaviour. A participant who moved from Ontario to Vancouver expressed his difficulty in adjusting to the weather. Ontario is a cold, but dry, region compared to Vancouver. Also, Vancouver has many microclimates because of the sea breezes. Vancouver weather is mild in winter compared to other Canadian cities. It is wet, and the weather keeps changing because of the seafront. Hence, it feels colder compared to Ontario weather. This participant felt colder in Vancouver than in Ontario, and his reason was Ontario weather is dry. The participant mentioned that he took long hot baths to regulate his body temperature. It took him a year to adjust to the regional climate. There were similar discussions when moving from one building to another building.

\section{Actionable knowledge is limited \\ "...for both of us thermostat up is the first thing to do!" $-D M$}

The research covers various issues in adaptation with controls like thermostat, blinds, doors or windows. 
The interaction with the thermostat is elaborated here, as the study shows it is the primary solution for discomfort. There might be other options, but action precedes reasoning. A few participants said they adjust the thermostat only in extreme weather or freezing room. Complex interface or technology (like a programmable thermostat) make the people passive with interactions. The other reason a person is passive is that they ignore their surroundings when focused on a task, for example, gaming. Participants also increase the thermostat for the following reasons: to reduce moisture or to avoid mold growth; conflict in comfort requirements; the presence of a baby, children, seniors or guests; people's sensitivity to cold; and finally if the inhabitant is doing an activity and is feeling cold. People need to understand that poor insulation, cooking, or showering may also cause an increase in moisture. Increasing the thermostat is one option to resolve the issues, but there are other solutions like improving the window insulation, ventilating the building sufficiently or use extractor fans. Awareness of possibilities and understanding building functions will affect their choice of actions. LWLA can demonstrate the possible criteria mentioned above, however it does not consider all possible variables, for example, LWLA does not provide suggestions to change building functions. Any system should consider the context to help inhabitants make a sustainability-related action. LWLA can help the inhabitants in weighing actions and making meaningful interactions. In conclusion, the needs of the inhabitants and interactions cannot be approached separately. They need to be approached holistically based on inhabitants' knowledge, awareness, memory, and comprehension.

\section{Understanding technology aids or obstructs interactions}

"...The only reason I would not change the thermostat .. is usually I find it unfriendly." $-M M$ Technology influences the daily practices of inhabitants. Most participants mentioned in a social context that it is "a constant battle" with the thermostat during conflicts over comfort issues. They often interact with the thermostat by raising or lowering it without reference to others. This leads to the question of how to use the thermostat efficiently to save energy. People do not understand how a thermostat influences the room temperature. For example, participants found it difficult to understand why the room was cold or hot after increasing or lowering the thermostat. Various factors may influence room temperature, and the inhabitants need to evaluate the causes to make appropriate changes to the building elements and controls. While understanding the function and use of technology is essential for adaptations, the real issue lies with the interface design of a control system, for example, the thermostat. Participants mentioned that they would not change the pro- grammable thermostat setting, as it is complicated to do so. One participant stated that he would like to express to the system "lower it for two hours." Voiceassisting systems like Alexa and Siri are becoming a part of daily life. The current generation is growing with technology and appreciates its use and incorporation. However, some people who are less technical may prefer to make their choices.

\section{Activities and body's core temperature ampli- fies interactions}

"...if I am doing physical work and am cold in here, I am more likely to turn the thermostat up"-IF

Inhabitants' activity is one of the core principles in an energy comfort model. These models use standard and scheduled assumptions. Activity affects the metabolism of the body: being inactive or sitting in a constant position for a long time will affect the body's core temperature. A few participants mentioned if they are working (like sewing or typing) and feeling cold they will increase the thermostat. According to Fanger's PMV model, if a person is working, then they are at specific temperature, and it is constant. However, the study shows that participants doing the work feel cold after some time. The PMV model considers the activity input as an immediate effect, but for most people, it is a gradual increase. A system can show the cause-effect of the actions.

\section{Barriers to sustainable adaptations}

The previous section explains the attributes from the inhabitants' perspective. Here, barriers represent the lack of information or interaction availability to them.

Agency

"You have no control"-EM \& EF

Agency must align with effect for a control system to have an impact. For example, if an inhabitant has no access to mechanical system controls, (s)he will resort to actions that yield comfort but may increase energy consumption. In a centrally heated apartment, the inhabitants have less control to operate for comfort. A couple mentioned that their building was too hot, and they had no control to change the settings. Instead, they adapted by wearing light clothes and walking barefoot in the apartment. No control leads to unwanted energy use. In an individual house that is partly sublet, the owner has more control over the thermostat setting than the tenants. A participant mentioned they were cold during winter and could not adjust the thermostat setting - so they are very uncomfortable. The participant also highlighted that the kitchen was cold as well. The owner preferred a different temperature setting. Lack of agency disrupts life.

\section{Knowledge of building performance}

"Turn on the gas fireplace, and then we crack open the windows for fresh air." - GM 
Most apartments for the study were well insulated and airtight, so overheating was an issue. For example, people use fans or leave windows open during winter when the room is over-heated. It is easy and fast to open a window; harder to turn down a thermostat and wait for the temperature to fall. LM mentioned he would like to know the effect of his action on energy usage and savings. LM, who was taking measures to save energy, became demotivated because he was not able to see its effect on cost. LWLA helps in this respect. The participant mentioned that he wished he could look at the data for his house after seeing the impact of different interactions in LWLA. Lack of information about energy usage hinders inhabitant awareness and motivation to take sustainable action. Most of the participants left all the windows and doors open during the summer. The operation may increase or decrease the temperature in the building, but, at particular times of the day and orientations of the building, operating windows or doors can be helpful for ventilation. The knowledge gap between system use and function affects interactions. For example, understanding the role of the thermostat, windows and blinds will change the way inhabitants interacting with them. Lowering a thermostat for overheating may or may not resolve the issue because sometimes it could be based on building design. Especially for apartments, heat is gained through stack effect and from shared walls. Most of the interactions performed by the inhabitants were energy consuming, and even during the task completion participants needed an explanation for specific actions. People can and will use qualitative performance explanations.

\section{Time latency}

"If I am feeling hot, I would probably open the door or a window. For at least a little bit.....turning the thermostat down will eventually take a long time"-LM

An action taken affects the future more than the present. Increasing the thermostat does not immediatey change room temperature. There is a time latency, and people do not understand it. They hesitate to both raise and lower the thermostat. The adaptations are mainly through windows or clothes as these effects are quickly felt. LM argues the room state is not changing but his metabolism is, so concludes by saying he will not lower the thermostat. Some activities take effect in the future and not at the current state. For example, initially, while reading a book the participant is at a comfortable room temperature. However, as time passes, the participant feels cold and increases the thermostat. In this case, the metabolism becomes low due to a steady state, hence drinking something warm or a short activity can change their metabolism. Participants need to understand the cause-and-effect of interactions and activity in the buildings.

\section{Trade-offs}

"What could I do to be comfortable and save economically?"-IF

Trade-off considers participant actions, where they choose adverse decisions like opening windows during winter instead of lowering the thermostat. Participants wear sweaters mostly as a trade-off or as a compromise during conflicts in comfortable room temperature. A practical design approach for the future is to consider both the parameters: comfort and energy use. One cannot be separated from the other. Most participants preferred one over the other, and few people gave equal preferences for both the parameters. Comfort typically takes priority, for example, a couple mentioned they like the building to be slightly cold, and hence they leave the doors open during winter. This couple also said that they do not care about energy use. Their reason was that gas is cheap. Likewise, people who have an economic constraint or if they are sustainability enthusiasts might trade a comfortable temperature. These may not be the healthiest interactions. While comfort was the most prominent influencer, aesthetics of the surrounding environment influenced as well. Making trade-offs clear and explicit may affect future choices.

\section{Adaptive impact of the proposed system}

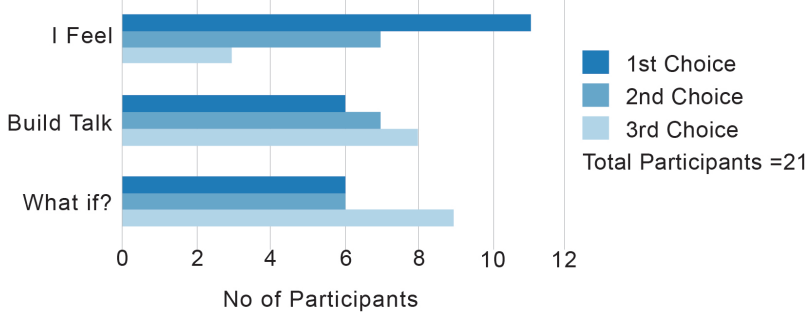

Figure 5: System dialogue in order of preference, Out of 21 responses, 11 chose "I feel". Two Participants chose "I feel" and "If buildings could talk" as equal first preferences (Rajus and Woodbury (2018)).

The "I feel" model was highly preferred compared to the other two models (See Figure 5) as it acknowledged people's sense of comfort and compared cost with room comfort. Figure 5 summarizes participants' preferences of the three models. "What if?" model was least preferred as it required more effort from participants. "If buildings could talk" was preferred by parents to notify them of their children's interaction. Participants said this model is helpful to learn their comfort and cost thresholds. Figure 6 gives an excerpt of comparing the three interaction models. The comparisons are detaily explained in the previous paper (Rajus and Woodbury (2018)).

\section{Decision-making}

"I think to look at this; it is sort of in your brain logical like 'close the door'."-HF Possible options and energy data helped participants to weigh the options and choose the action. During 


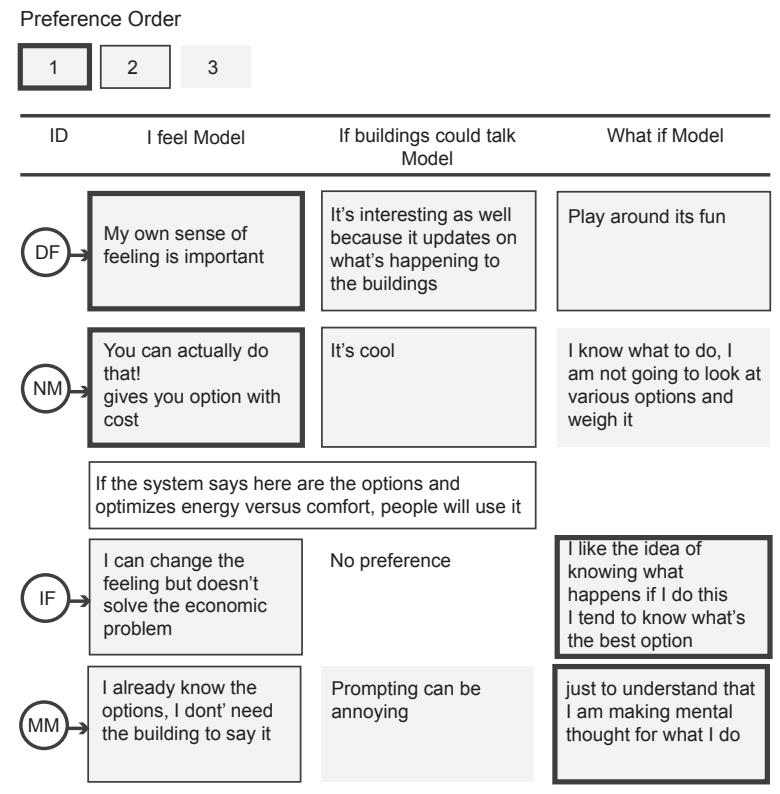

Figure 6: Excerpt of Model comparison of the three interaction models (Rajus and Woodbury (2018)).

the pre-questionnaire, some participants' initial interaction was to increase the thermostat when the building was cold and leave the windows open for fresh air. However, on seeing the information, they understood its impact and changed their choices. They were willing to make combined actions like lowering thermostat and wearing a sweater. Lowering the thermostat was not a standard response to the prequestionnaire, but during the task completion, when participants saw the comparative data, they said they would choose to lower thermostat if it saves some cost.

\section{Learning}

"in that absence of that information, all we know is that it makes us have a better sleep"-LF

During the study, participants learned the effect of their actions on why one choice was more efficient than the other. For example, instead of increasing the thermostat, a participant chooses to wear a sweater. This action brought them to a comfortable perceived temperature. But they also noticed that lowering the thermostat by 2 degrees and wearing a sweater causes low energy usage and gives the same comfort. There were questions about personal changes like wearing a sweater and its effect on energy usage. The sweater does not affect energy usage directly but is a sustainable action. They wondered why lowering the thermostat reduces the cost and the sustainable action of wearing a sweater does not. Here participants need to see the effect of their actions. So, seeing the options helped them in narrowing their choice. Also, one participant mentioned that "I feel" and "If buildings could talk" will help children to learn why one option is better than others. Participants note that, while "I feel" is easy to express, "If buildings could talk" could help them learn about comfort thresholds and options.

\section{Negotiation}

"Actually this would help us to reach probably the collective agreement"-DM

Most of the couples mention during the prequestionnaire that differing comfort requirements created a "constant battle" or a need to compromise on differing comfort requirements. While conducting the task completion, I noticed that the participants started consulting with each other on the options and what choice they would like to make. They discussed and weighed the options and came to a conclusion. In fact, one of the participants stated that LWLA or a system like this would help them in negotiation.

\section{Trust and control}

"I am initializing it" $-P M$

Participants mention that they trusted the system because they are in control of decisions made. Across the three interaction models, they initiate actions and make choices. The system only provides or suggests possible comfort and energy options. Participants trusted the system because the decision was initiated and concluded by them. A few participants suggested partial automation and system intelligence to learn their preferences, but expressed they would still like to be in control. In LWLA, the inhabitant controls and initiates action.

\section{Conclusion}

The intent of LWLA is to close the gap between design intent and actual use of the building by providing information on and rich interactive control of comfort and energy usage. The present study, which presented LWLA to a variety of households, expanded our understanding of providing effective environmental controls. From an inhabitant's perspective, it shows that background, culture, knowledge and activity all affect interactions within buildings. From a system perspective, it shows a rich variety of barriers and opportunities that inform design of future interactions. The LWLA prototype itself is only a start. While its commitment to structuring interactions around human-system "conversations" (e.g., "I feel") shows promise, the study revealed many other questions for interaction. For instance, LWLA was designed with the implicit assumption of being controlled by a single person, whereas the study showed that active negotiation is a real part of family interaction with the building system.

\section{Towards a future LWLA}

LWLA considers only thermal comfort in its system design. Often the actions of inhabitants affect other energy resources. For example, closing thermal blinds may reduce heat loss but affects daylighting. A future system may consider other comfort parameters like lighting and ventilation. Each of the barriers listed in the Findings section poses new design challenges. Perhaps the most difficult, and therefore the first that 
should be addressed, is the fact that interaction is mostly a group, rather than an individual, activity. While we believe that LWLA's conversation-based design has strong potential, we argue that some of the barriers be better addressed before LWLA is implemented and longitudinally evaluated in residences.

\section{Acknowledgments}

We gratefully acknowledge the support of the Pacific Institute of Climate Solutions, the NSERC Discovery and CRD programs, and Bentley Systems, Inc.

\section{References}

Abrahamse, W., L. Steg, C. Vlek, and T. Rothengatter (2005). A review of intervention studies aimed at household energy conservation. Journal of Environmental Psychology 25(3), 273 - 291.

Bartram, L., J. Rodgers, and R. Woodbury (2011). Smart homes or smart occupants? supporting aware living in the home. In HumanComputer Interaction-INTERACT 2011, pp. 5264. Springer.

Becker, R. and M. Paciuk (2009, May). Thermal comfort in residential buildings - failure to predict by standard model. Building and Environment 44(5), 948-960.

Cole, R. and Z. Brown (2009). Reconciling human and automated intelligence in the provision of occupant comfort. Intelligent buildings international 1(1), 39-55.

Cole, R. J., Z. Brown, and S. McKay (2010). Building human agency: a timely manifesto. Building Research \& Information 38(3), 339-350.

Darby, S. (2006). The effectiveness of feedback on energy consumption: A review for DEFRA of the literature on metering, billing and direct displays. Environmental Change Institute, University of Oxford.

De Dear, R. (2004). Thermal comfort in practice. Indoor Air 14, 32-39.

De Wilde, P. (2014). The gap between predicted and measured energy performance of buildings: A framework for investigation. Automation in Construction 41, 40-49.

Fabi, V., G. Spigliantini, and S. P. Corgnati (2017). Insights on smart home concept and occupants' interaction with building controls. Energy Procedia 111, 759 - 769. 8th International Conference on Sustainability in Energy and Buildings, SEB-16, 11-13 September 2016, Turin, Italy.

Faruqui, A., S. Sergici, and A. Sharif (2010). The impact of informational feedback on energy consumption: A survey of the experimental evidence. Energy 35(4), 1598-1608.

Flemming, S. A., A. Hilliard, and G. A. Jamieson (2008). The need for human factors in the sus- tainability domain. In Proceedings of the Human Factors and Ergonomics Society Annual Meeting, Volume 52, pp. 748-752. SAGE Publications.

Froehlich, J., L. Findlater, and J. Landay (2010). The design of eco-feedback technology. In Proceedings of the 28th international conference on Human factors in computing systems, pp. 1999-2008. ACM.

Hampton, A. (2011). Designing user-friendly passive buildings. Environment Design Guide, Royal Australian Institute of Architects (EDG 67 AH), 1-10.

Hoes, P., J. Hensen, M. Loomans, B. De Vries, and D. Bourgeois (2009). User behavior in whole building simulation. Energy and buildings 41(3), 295302 .

Humphreys, M. A. and J. Fergus Nicol (2002). The validity of iso-pmv for predicting comfort votes in every-day thermal environments. Energy and buildings 34(6), 667-684.

Jia, M., R. S. Srinivasan, and A. A. Raheem (2017). From occupancy to occupant behavior: An analytical survey of data acquisition technologies, modeling methodologies and simulation coupling mechanisms for building energy efficiency. Renewable and Sustainable Energy Reviews 68, 525-540.

Lomas, K. J. and S. M. Porritt (2017). Overheating in buildings: lessons from research. Building Research E Information 45(1-2), 1-18.

Majcen, D., L. Itard, and H. Visscher (2013). Theoretical vs. actual energy consumption of labelled dwellings in the netherlands: Discrepancies and policy implications. Energy policy 54, 125-136.

Mozer, M., R. Dodier, D. Miller, M. Anderson, J. Anderson, D. Bertini, M. Bronder, M. Colagrosso, R. Cruickshank, B. Daugherty, et al. (2005). The adaptive house. In IEE Seminar Digests, Volume 11059, pp. v1-39. IET.

Nord, N., T. Tereshchenko, L. H. Qvistgaard, and I. S. Tryggestad (2018). Influence of occupant behavior and operation on performance of a residential zero emission building in norway. Energy and Buildings 159, 75-88.

Rajus, V. S. and R. Woodbury (2018). Light weight look ahead options for personal interaction in buildings. In Proceedings of the Symposium on Simulation for Architecture and Urban Design, pp. 13. Society for Computer Simulation International.

Strengers, Y. (2008). Challenging comfort and cleanliness norms through interactive in-home feedback systems. In Pervasive 2008 Workshop Proceedings, pp. 104-108.

Woodruff, A., S. Augustin, and B. Foucault (2007). Sabbath day home automation: it's like mixing technology and religion. In Proceedings of the SIGCHI conference on Human factors in computing systems, pp. 527-536. ACM. 